\title{
Geschichte des Botanischen Gartens an der Siesmayerstraße in Frankfurt am Main
}

\author{
Georg Zizka, Stefan Dressler und Manfred Wessel
}

\begin{abstract}
The history of Botanical Gardens in Frankfurt dates back to 1763, when Johann Christian Senckenberg founded his "Dr. Senckenbergische Stiftung", which established the first Botanical Garden of Frankfurt and founded the garden for more than 150 years. Then, the Goethe University was responsible for the Botanical Garden for almost a hundred years. Since January 1st, 2012, the Botanical Garden Frankfurt belongs to the City of Frankfurt. In this long history there have been several relocations, reductions and enlargements of the area, far-reaching changes in content and finally even the setup of another Botanical Garden ("Wissenschaftsgarten") by the University. The history of the three Botanical Gardens in Frankfurt is a good example of the importance of civic engagement and the close connection between private and public scientific institutions and associations in Frankfurt.
\end{abstract}

\section{Zusammenfassung}

Der Botanische Garten an der Siesmayerstraße in Frankfurt ist seit dem 1.1.2012 Teil des städtischen Palmengartens. Die Geschichte eines Botanischen Gartens in Frankfurt reicht bis ins Jahr 1763 zurück, als Johann Christian Senckenberg die Dr. Senckenbergische Stiftung begründete, die dann Erbauerin und über 150 Jahre Trägerin des ersten Frankfurter Botanischen Gartens war. Fast hundert Jahre war dann die Goethe-Universität für den Botanischen Garten verantwortlich, heute ist es die Stadt Frankfurt. In dieser langen Geschichte ist es zu mehreren räumlichen Verlagerungen, Verkleinerungen und Vergrößerungen des Areals, tiefgreifender inhaltlicher Umgestaltung und schließlich sogar zur Entstehung eines weiteren Botanischen Gartens, des Wissenschaftsgartens am Riedberg, gekommen. Die Geschichte der Botanischen Gärten in Frankfurt ist ein gutes Beispiel für die große Bedeutung von Bürgerengagement und die enge Verbindung von privaten und öffentlichen wissenschaftlichen Institutionen und Vereinen in Frankfurt.

\section{Der erste Botanische Garten in Frankfurt am Main}

Die ersten Gründungen von Botanischen Gärten in Europa erfolgten bereits im 16. Jahrhundert an Universitäten zur Ausbildung der Mediziner in der Heilpflanzenkunde (Padua 1545, Leipzig ca. 1580, Leiden 1590, Heidelberg, 1593).

Deutlich später, aber ebenfalls mit dem Ziel der Medizinerausbildung, wurde der erste Botanische Garten in Frankfurt als Teil der Dr. Senckenbergischen Stiftung gegründet. Der Frankfurter Arzt Johann Christian Senckenberg (28.2.1707-15.11.1772) begründete mit seinem Vermögen die nach ihm benannte Stiftung, am 18.8.1763 unterzeichnete er den Hauptstiftungsbrief. Sein Ziel war es, die Medizinerausbildung und die medizinische Versorgung in Frankfurt zu verbessern und die Naturwissenschaften allgemein zu fördern. Die Dr. Senckenbergische Stiftung erwarb 1766 ein etwa 2,8 ha großes Grundstück am Eschenheimer Turm und begann dort mit der Anlage des "Senckenbergianums“, das zunächst aus einem Krankenhaus für die Frankfurter Bürger sowie Einrichtungen für die Mediziner- ausbildung wie Anatomie-Hörsaal, Bibliothek und einem Botanischen Garten („Medizinalgarten“; Abb. 1) bestehen sollte. 1767 wurde als erster „Stiftsgärtner“ JohanN HeInRICH BÄUMERTH (7.1.1743-25.4.1816) eingestellt, 1773 der erste "Stiftsarzt" und gleichzeitig Direktor des Botanischen Gartens, Johann Jаков ReICHARD (7.8.1743-21.1.1782). BäUMERTH und Senckenberg planten den Garten gemeinsam nach dem Vorbild des Botanischen Gartens in Uppsala (Abb. 1). Zunächst wurde 1768 ein 18 x 7,7 m großes Gewächshaus fertiggestellt. Die Anlage des Freilandbereiches erfolgte ab 1774, also nach dem Tode Senckenbergs, unter der Leitung von Reichard und BäUmerth. Die erste gedruckte Artenliste des Gartens von 1782 umfasste bereits beeindruckende 1430 Arten, die zwischen 1777 und $1781 \mathrm{im}$ Garten kultiviert worden waren (ReICHARD 1782). Wie von SENCKENBERG gefordert, lag der Schwerpunkt auf einheimischen und aus vergleichbaren Klimaten stammenden Gewächsen, insbesondere Arzneipflanzen.

Rund 100 Jahre lang folgten Mediziner als Gartendirektoren und Stiftsärzte auf den früh ver- 


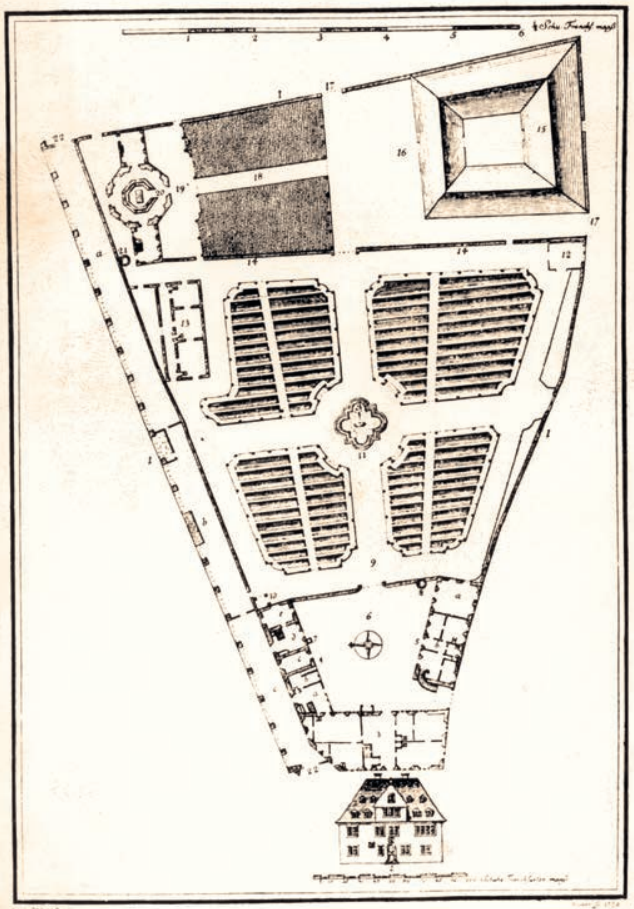

Abb. 1: Plan des Dr. Senckenbergischen Stiftungsgeländes mit der vorgesehenen Gartenanlage (1770). Die Beete des Medizinalgartens (9) sind - wie damals üblich - regelmäßig gestaltet, die Anordnung der Pflanzen erfolgte nach dem LINNÉ'schen System. Das etwa $140 \mathrm{~m}^{2}$ große, dreiteilige Gewächshaus (13) war schon 1768, vor der Anlage des Gartens, fertiggestellt worden.

storbenen Reichard. An der inhaltlichen Ausrichtung des Gartens änderte sich in dieser Zeit wenig. Mit der von der Dr. Senckenbergischen Stiftung maßgeblich mitgetragenen Gründung der Senckenbergischen Naturforschenden Gesellschaft (SNG, seit 2008 Senckenberg Gesellschaft für Naturforschung, SGN) im Jahre 1817 gewannen die Naturwissenschaften gegenüber der Medizin stärkere Beachtung. Die ehrenamtlich tätigen Sektionäre (Betreuer) der botanischen Sammlungen der SNG waren in der Regel im Brotberuf Beschäftigte der Dr. Senckenbergischen Stiftung. So z. B. JoHANNES BECKER (20.2.1769-24.11.1833), der ab 1817 Stiftsgärtner der Dr. Senckenbergischen Stiftung und Begründer der botanischen Sammlungen der SNG war. Dies erklärt, warum sich im Herbarium Senckenbergianum der SNG aus dieser Zeit viele Belege von Gartenpflanzen aus dem Botanischen Garten finden. Hermann Theodor Ge-

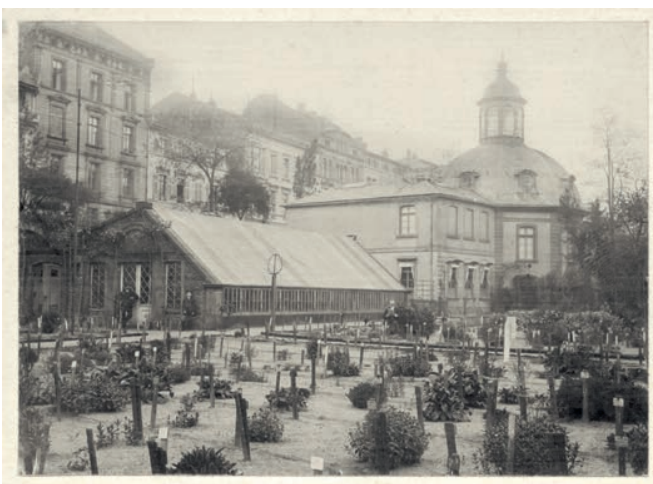

Abb. 2: Der erste Botanische Garten im alten „Senckenbergianum" am Eschenheimer Turm, Blick vom Zentrum des Gartens nach Nordosten. In der Bildmitte sind das (zweite) Gewächshaus und dahinter die Senckenbergische Anatomie zu erkennen. (Rittweger 1905)

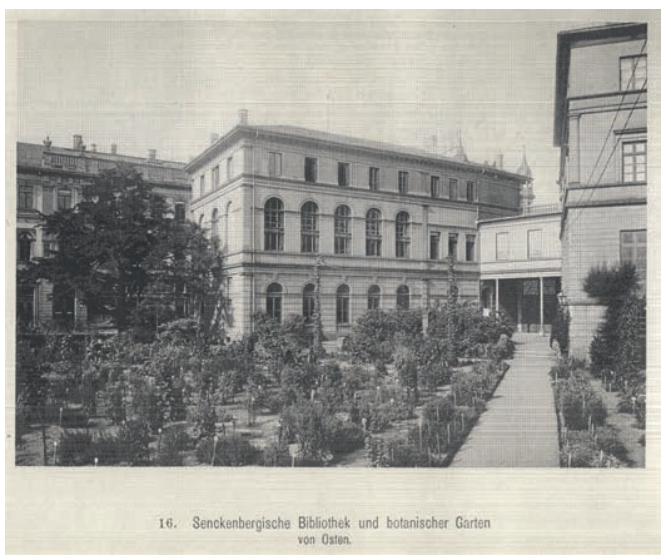

Abb. 3: Der erste Botanische Garten im alten „Senckenbergianum“ am Eschenheimer Turm (um 1900), Blick vom Zentrum des Gartens nach Südwesten. Ganz links im Bild die später verpflanzte Alte Eibe, rechts daneben die Senckenbergische Bibliothek, daneben der Eingang zum Senckenbergianum vom Eschenheimer Turm her und ganz rechts das Senckenberg-Museum. (Rittweger 1905)

YLER (15.6.1834-22.3.1889) war dann der erste „echte“ Botaniker, der Direktor des Botanischen Gartens wurde. Zu seiner Zeit sollen über 4000 Pflanzenarten im Garten kultiviert worden sein.

Bereits 1850 erfolgte eine Vergrößerung des Gartens bis zur Brönnerstraße. Die Erweiterung des Bürgerhospitals und der Neubau der Gebäude für den 1824 gegründeten Physikalischen Verein, die Dr. Senckenbergische Bibliothek sowie für das Senckenberg-Museum gingen aber zu Lasten der 


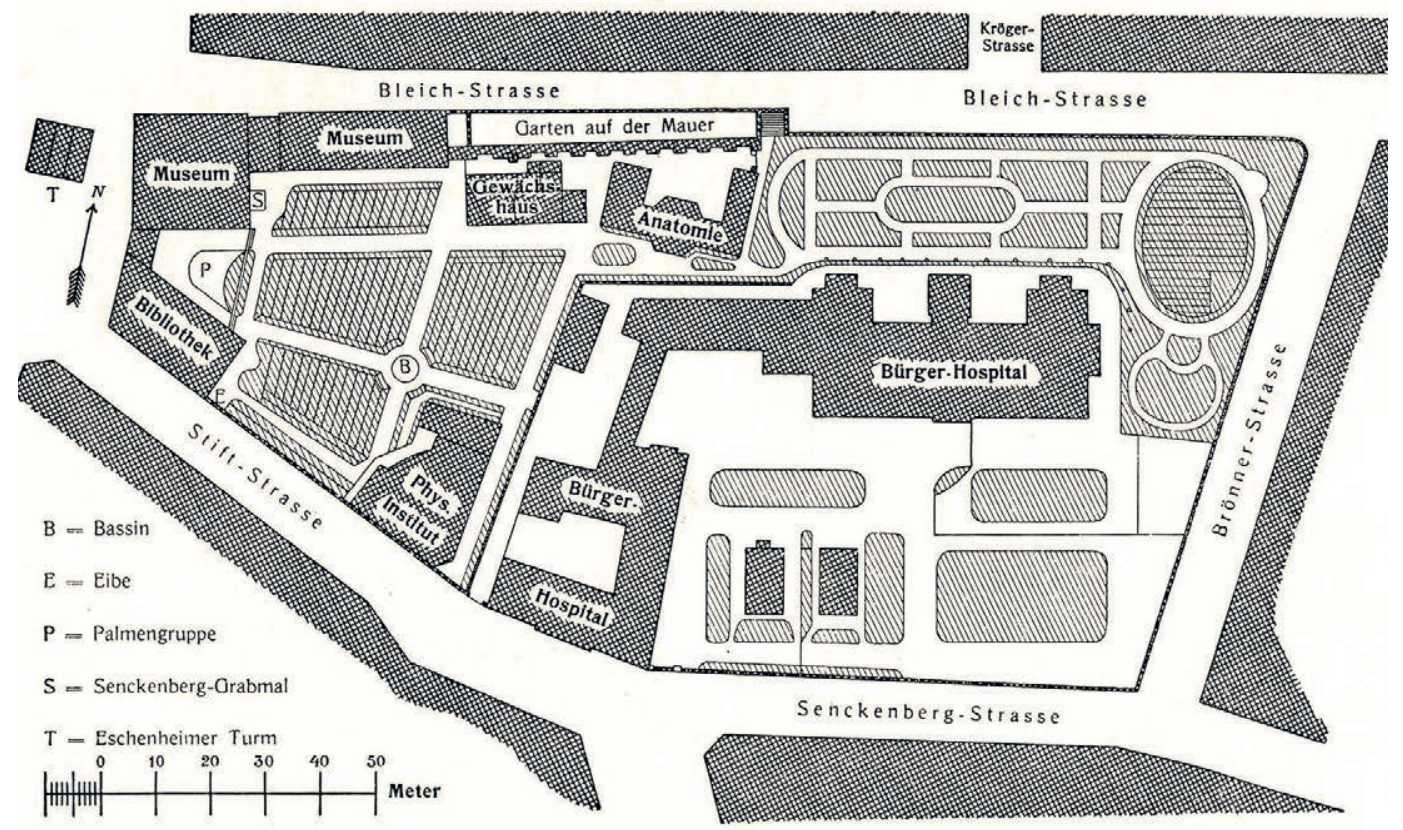

Bild 3: Grundriß der Anlagen und Bauten der Senckenbergiscben Stiftung, der Senckenbergischen Naturforschenden Gesellschaft und des Physikalischen Vereins am Ende des 19. Jabrbunderts. Nach M. Möbius (22).

Abb. 4: Plan des ersten Botanischen Gartens am Eschenheimer Turm am Ende des 19. Jahrhunderts. (MöвIUs 1903)

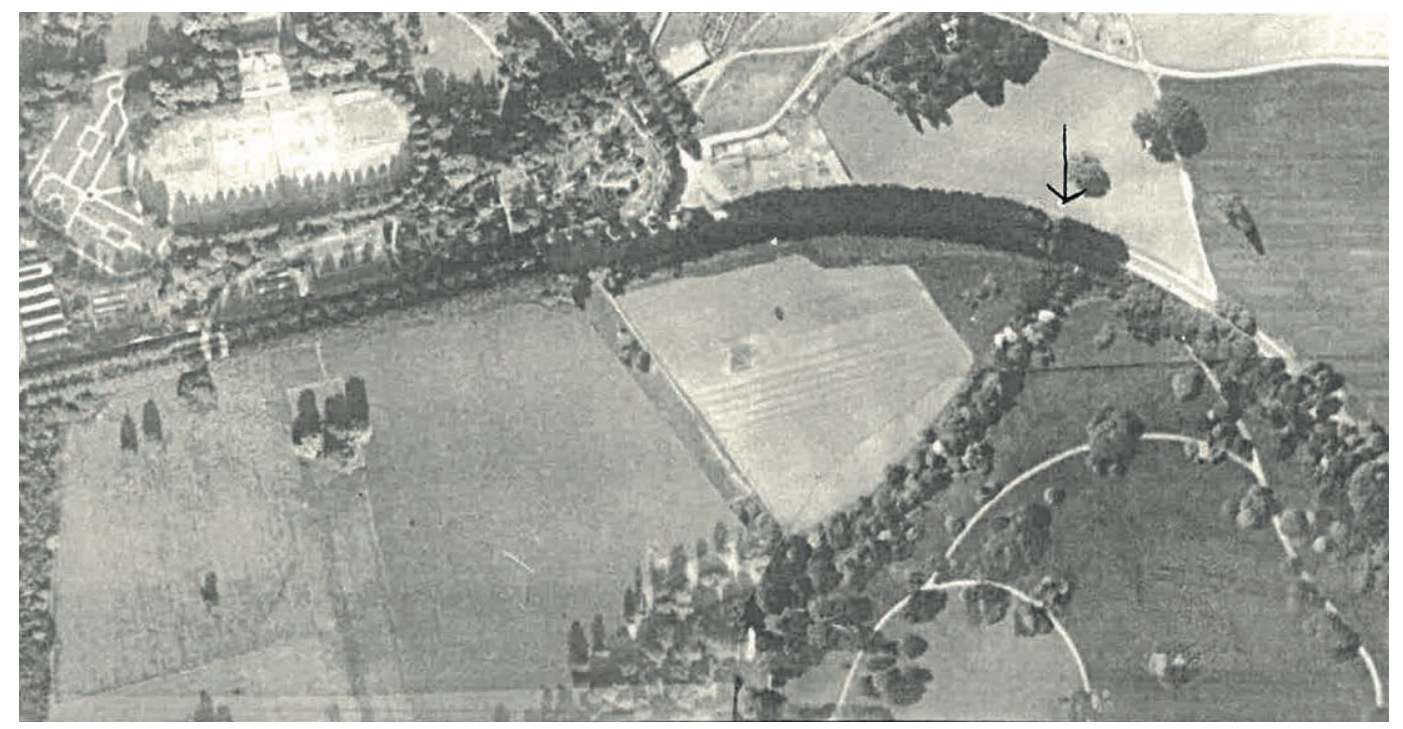

Abb. 5: Ausschnitt aus einem Luftbild der Stadt Frankfurt von 1926/27. Im linken oberen Bildteil ist der nordöstliche Teil des Palmengartens zu erkennen (am linken Bildrand das damalige Tropen-Schauhaus, dessen Mittelteil in den 1980er-Jahren um $90^{\circ}$ gedreht wurde und heute das Eingangsschauhaus bildet). Die helle elliptische Fläche ist das Areal des Tennisclubs Palmengarten, der 1989-1992 an den Berkersheimer Weg verlegt wurde. (Foto: Südwestdeutsche Luftverkehrs A.G., Frankfurt, Institut für Stadtgeschichte Frankfurt am Main) 

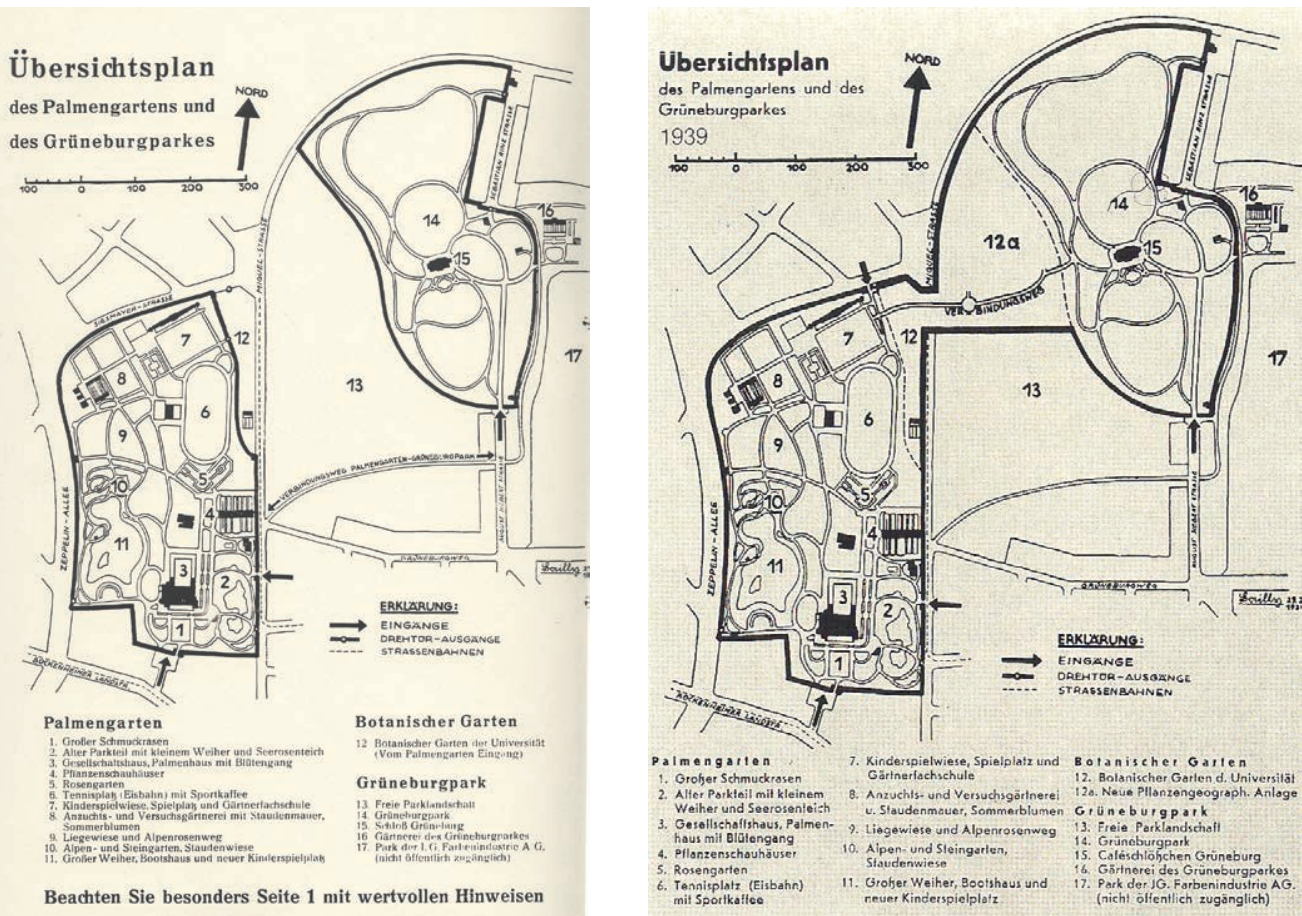

Abb. 6: Plan des Palmengartens und des Zweiten Botanischen Gartens aus der Zeitschrift „Der Palmengarten, links von 1936, rechts von 1939. Auf dem Gelände des Botanischen Gartens ist das Gewächshaus eingezeichnet (s. auch Abb. 7). Das Gelände des dritten Gartens ist links noch Teil des als „Freie Parklandschaft“ (13) bezeichneten Gebietes. Einige Jahre später haben die Arbeiten am dritten Garten offensichtlich schon begonnen, das Gelände ist als „Neue pflanzengeographische Anlage (12a) ausgewiesen.

Gartenfläche, die gegen Ende des 19. Jahrhunderts nur noch ca. 0,7 ha betrug. Die fehlenden Erweiterungsmöglichkeiten führten schließlich Anfang des 20. Jahrhunderts zu einer Aufteilung und Verlagerung der Senckenbergischen Institute. Der Botanische Garten zog 1907/1908 auf ein etwa 1,4 ha großes Gelände am nordöstlichen Rand des Palmengartens um, das die Stiftung von der Familie Rothschild erwerben konnte (Abb. 5 und 6). Zum Umzug gehörte auch die aufwändige Verpflanzung der „Alten Eibe“ 1907, die ganz Frankfurt für mehrere Tage in Atem hielt. Eine detaillierte Beschreibung der Verpflanzungsaktion, die etwa so teuer wie die Anlage des restlichen Gartens gewesen sein soll, findet sich bei BLECKEN (1994, 1995).

\section{Der zweite Botanische Garten}

Seit 1893 war Dr. Martin Möвıus (7.12.185925.1.1946) als Angestellter der Dr. Senckenbergischen Stiftung Direktor des Botanischen Gar- tens und „2. Bibliothekar“. Bereits 1899 legte МӧвıUs der Stiftungsadministration seine Pläne zur Zukunft des Botanischen Gartens in einem vierseitigen Brief dar. МӧвıUs und Obergärtner Rudolph GüNther planten und leiteten die Realisierung des Gartens am neuen Standort (s. Abb. 5 und 6). In Personalunion hatte Möвıus auch die ehrenamtliche Funktion des „Sektionärs für Botanik" der SNG inne. Als 1914 die Frankfurter Universität gegründet wurde, bildeten Dr. Senckenbergische Stiftung, SNG und Physikalischer Verein den Grundstock der Naturwissenschaften. Der Botanische Garten wurde Teil der Universität, Mӧвıus der erste Professor für Botanik. Er nahm also in Personalunion an allen drei Institutionen Aufgaben wahr und repräsentierte die enge Vernetzung, die mit dem Dienstantritt seiner Nachfolger Peter Stark (5.8.1888-9.11.1932) im Jahre 1928 und danach Friedrich Laibach (2.4.1885-5.6.1967) im Jahre 1934 endete. Für die mit Gründung der Universität erheblich ge- 


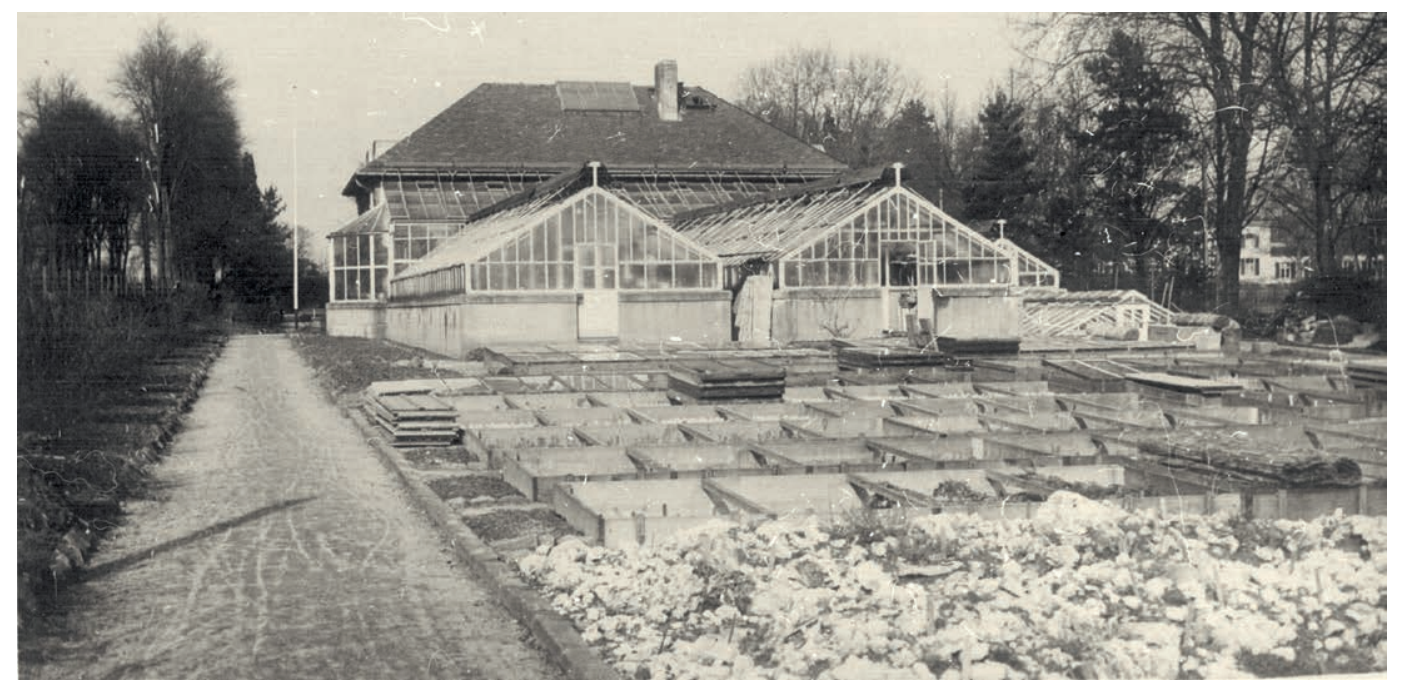

Abb. 7: Frühbeetkästen, Gewächshäuser und Betriebsgebäude im Südteil des zweiten Botanischen Gartens (Blickrichtung nach Norden). Beim nur undeutlich zu erkennenden Gebäude am linken Bildrand muss es sich um das Haus Leonhardtsbrunn im Palmengarten handeln. (Foto: Karl Reis, aus Egle \& Rosenstock 1966)

wachsenen Aufgaben musste eine entsprechende Infrastruktur geschaffen werden. Das neu entstandene Botanische Institut, zu Beginn noch wesentlich getragen von der Dr. Senckenbergischen Stiftung, fand schließlich im östlichsten Teil des JüGEL-Hauses (damaliges Hauptgebäude der Goethe-Universität), über der im Erdgeschoss untergebrachten Dr. Senckenbergischen Bibliothek, sein Zuhause.

Der mit der Aufgabe des Senckenbergianums am Eschenheimer Tor neu angelegte zweite Botanische Garten bestand aus der systematischen Abteilung, Alpinum sowie weiteren kleineren Revieren (BuCHER 1998). Im südlichen Gartenteil entstand 1914 eine Gewächshausanlage mit rund $400 \mathrm{~m}^{2}$ unter Glas (s. Abb. 7).

\section{Der dritte Botanische Garten}

Der zweite Botanische Garten erwies sich allerdings schon nach zwei Jahrzehnten erneut als zu klein für die Anforderungen von Forschung und Lehre. Erschwerend kam hinzu, dass seine thematische Ausrichtung nicht mehr den damaligen modernen Forschungsrichtungen entsprach, der Pflanzengeographie, -ökologie und -genetik. So wurde eine Verlagerung auf ein angrenzendes Areal, eine Vergrößerung auf rund 7,5 ha und eine inhaltliche Erneuerung in Angriff genommen. Zunächst wurde das Gelände gepachtet, 1935 erwarb es die Stadt Frankfurt als Teil des Grüneburgpark-Geländes vom damaligen Eigentümer Albert von Goldschmidt-Rothschild (3.6.1879-26.12.1941). Dieser unter den damaligen Umständen erzwungene „Verkauf“ gegen eine wohl geringe Gegenleistung wurde von den Nachkommen Goldschmidt-Rothschilds nach dem Krieg angefochten und vom Gericht 1951 als unrechtmäßig bestätigt. In einem Vergleich erhielt die Familie als Entschädigung andere Grundstücke in Frankfurt und einen Geldbetrag und erklärte sich mit dem Verbleib des Grüneburgparks und des Gartengeländes im Besitz der Stadt Frankfurt einverstanden (Mertens 2014). Das spätere Gartengelände war Teil des Parks, und die Stadt stellte es der Dr. Senckenbergischen Stiftung im Tausch gegen das Areal des zweiten Botanischen Gartens (1,4 ha) zur Verfügung. Der frei werdende südliche Teil des zweiten Botanischen Gartens wurde Teil des Palmengartens, der nördliche Teil letztendlich Bestandteil des dritten Botanischen Gartens.

Die Planungen für den dritten Botanischen Garten hatten bereits in den 1930er Jahren begonnen, ab 1936 erfolgte die Gestaltung der Anlage (Egle \& Rosenstock 1966). Auch bei der 
Konzeption des neuen Gartens spielte die Nähe des Palmengartens mit seinen großen, öffentlich zugänglichen Gewächshausanlagen und großen tropischen Sammlungen eine Rolle Die Gewächshauser des Botanischen Gartens wurden daher klein und ausschließlich im Hinblick auf den Bedarf für Forschung und Lehre konzipiert. Für Planung und Realisierung waren der Technische Leiter Kurt Kiehne (15.7.1895-3.9.1990) und die aufeinander folgenden Garten-Direktoren Friedrich Laibach, Camill MontFORT (9.2.1890-19.9.1956), Wilhelm Halbsguth (14.8.1912-21.5.1988) und Karl Egle (15.9.1912-26.10.1975) verantwortlich. Anders als bei den beiden ersten Gärten bestand die neue Anlage zum großen Teil aus nach pflanzengeographisch-ökologischen Gesichtspunkten gestalteten Revieren (z. B. Buchenwald und andere mitteleuropäische Waldtypen, atlantische Zwergstrauchheide, Glatthaferwiese, Ostasien, Nordamerika, Mittelmeer, Kaukasus, Alpinum s. Alberternst et al. 2005, Jenny et al. 2014). Als für die Lehre unverzichtbarer Teil entstand wieder eine umfangreiche systematische Abteilung mit angrenzenden Lehrpflanzungen zur Züchtungsgeschichte wichtiger Kulturpflanzen. Die 1965 fertiggestellten Gewächshäuser boten nun die Gelegenheit, die Lehr- und Forschungssammlung tropischer Pflanzen angemessen zu kultivieren und auszubauen.

Das Areal war zu Beginn der Bauarbeiten größtenteils baumlos (Abb. 8 bis 10). Erst nach langer Unterbrechung durch Krieg und anschließende Sperrung des Geländes wegen der Nutzung für die alliierten Streitkräfte konnte der Garten ab 1948 mit der Gestaltung der nordamerikanischen und ostasiatischen Abteilung, des Alpinums sowie der Arzneipflanzenabteilung (1955-1957) weiter aufgebaut werden. 19611965 wurden die Gewächshausanlage, das Laborgebäude und das Wirtschaftsgebäude des Gartens errichtet. Diese Bauarbeiten wurden unter der Regie von Gartenamtsrat Herbert Becela (16.04.1927-30.07.2016) abgeschlossen, der als Nachfolger von KuRT KieHne (15.07.189503.09.1990) von 1960 bis 1992 Technischer Leiter des Botanischen Gartens war. Die Geschichte des

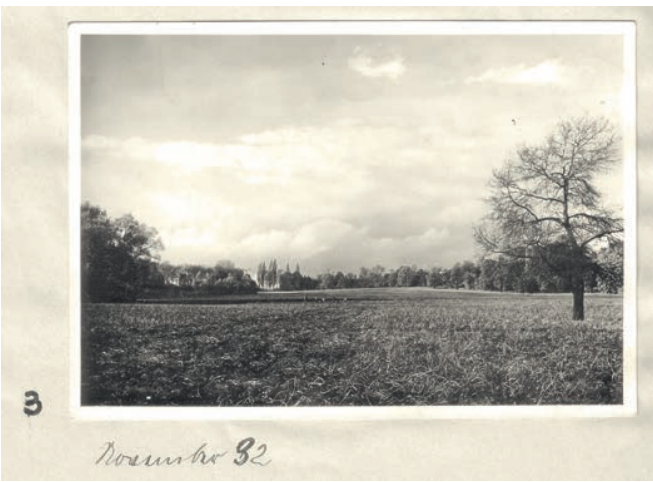

Abb. 8: Blick vom Süden auf die zukünftige Fläche des dritten Botanischen Gartens im Jahre 1932. (Archiv Abteilung Botanik und Molekulare Evolutionsforschung, Senckenberg Frankfurt)

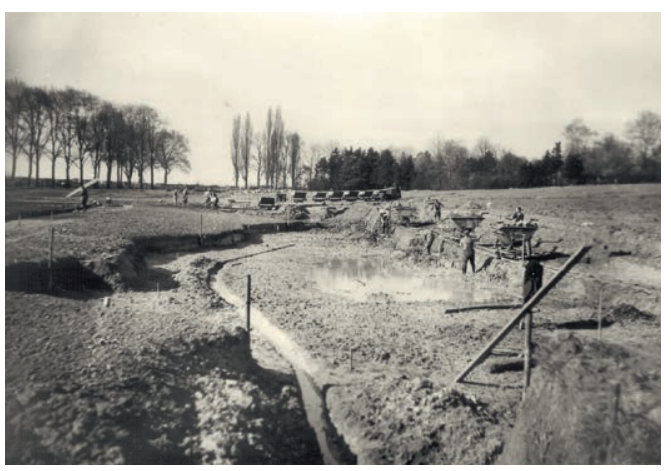

Abb. 9: Erdarbeiten für die Anlage des großen Weihers im dritten Botanischen Garten. Das Areal des zukünftigen Gartens ist 1939 praktisch vegetationslos. Die Baumreihe am linken Bildrand steht entlang der Verlängerung der Siesmayerstraße, die dann später geschlossen wurde; damals hieß sie Miquelstraße. (Archiv Abteilung Botanik und Molekulare Evolutionsforschung, Senckenberg Frankfurt)

zweiten Botanischen Gartens verlief also längere Zeit parallel zu der des dritten (1936-1964), bedingt durch dessen lange Baugeschichte. Die Geschichte des zweiten Gartens endet erst mit der Übernahme dessen südlicher Hälfte durch den Palmengarten 1964. Die von Universitätsbaudirektor Ferdinand Kramer geplanten Gebäude der Biologischen Institute entstanden bereits ab 1954. Von Seiten der Biologie betreute Prof. Dr. Günther Rosenstock (13.1.1922-3.10.1996) die Neubauten (Zizka 2005).

Als Ordinarius (Lehrstuhlinhaber) war Prof. Dr. Karl Egle seit 1.4.1959 Direktor des Botani- 


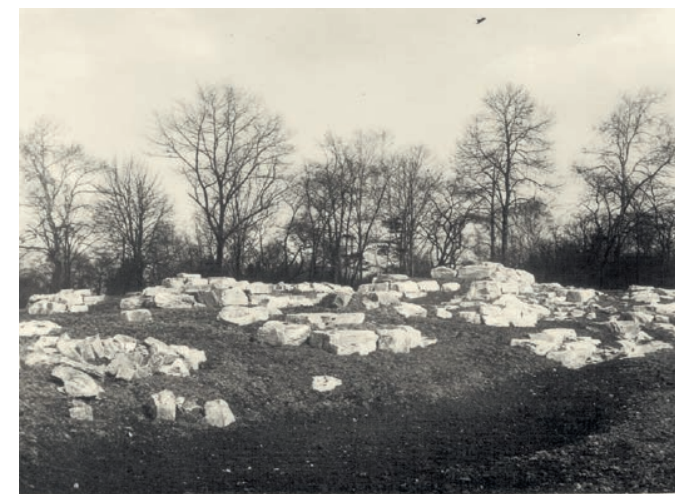

Abb. 10: Beginn der Anlage des Alpinums 1939. Im Hintergrund Baumbestand des Grüneburgparkes. (Archiv Abteilung Botanik und Molekulare Evolutionsforschung, Senckenberg Frankfurt)

schen Gartens und des Botanischen Instituts. Unter EGLE entwickelten sich sehr gute Beziehungen zwischen Palmengarten und Botanischem Garten und Institut, er etablierte auch regelmäßige Lehrveranstaltungen zu Nutz- und Zierpflanzen im Palmengarten, die nach Egles Tod von Prof. Dr. Wilhelm Lötschert (7.11.1923-29.6.1984) und Prof. Dr. Horst Lange-Bertalot weitergeführt wurden. Der Palmengarten-Direktor Prof. Dr. Gustav Schoser (22.8.1924-5.1.2012) benannte 1975 ein (heute nicht mehr vorhandenes) Gewächshaus im Palmengarten nach ihm (KARLEgle-Haus), das im Bereich des heutigen Rosengartens stand. Mit der Reformierung der hessischen Universitäten 1970 und der Abschaffung der Ordinariate lag die Direktion des Botanischem Gartens und Botanischen Institutes in Frankfurt bei einem jährlich aus der Professorenschaft gewählten „Geschäftsführenden Direktor" (Direktorinnen gab es bis zur Auflösung des Instituts keine). Dies führte zu oft jährlich wechselnden Direktoraten und - auf Grund der unterschiedlichen Forschungsrichtungen - unterschiedlich engen inhaltlichen Bezügen zwischen Direktor und Garten. Das war ein Unterschied zu den meisten anderen universitären Gärten, bei denen die Direktion des Gartens mit einer fachlich besonders nahestehenden Professur dauerhaft verbunden ist. Eine weitere Frankfurter Besonderheit war das Fehlen der Stelle eines „Gartenkustos“, also eines speziell mit den Pflanzensammlungen befassten Wissenschaftlers. Mit der Auflösung des
Botanischen Instituts am 30.9.2005 im Zuge der Neuordnung des Fachbereichs gelangte der Botanische Garten in die unmittelbare Zuständigkeit des Dekanats des Fachbereichs Biologie (ab 2005 Fachbereich Biowissenschaften). Die inhaltliche Leitung des Gartens war seit den 1990er-Jahren vom Geschäftsführendem Direktor, später DekanIn an die "Gartenkommission“ bzw. deren Leiter delegiert, die aus den fachlich intensiv mit dem Garten befassten Professuren und dem Technischen Leiter des Gartens und seinem Stellvertreter bzw. später Stellvertreterin bestand.

\section{Der dritte Botanische Garten wird städtisch}

Der Botanische Garten und das Botanische Institut der Goethe-Universität an der Siesmayerstraße lagen seit 1956 in unmittelbarer räumlicher Nachbarschaft und boten damit ideale Arbeitsbedingungen für Forschung und Lehre. Anders als der benachbarte Palmengarten mit seinen Hunderttausenden von Besuchern „blühte“ der Botanische Garten allerdings aus Sicht der außeruniversitären Öffentlichkeit lange Zeit im Verborgenen. Die Besucherzahlen waren bis in die 1980er-Jahre gering. Wie auch andere Universitätsgärten begann der Botanische Garten erst ab Anfang der 1990er-Jahre sich auch zusätzlich für eine breitere Öffentlichkeit zu öffnen. Andere Veränderungen zeichneten sich bereits in den 1970er-Jahren infolge des Wachstums der Goethe-Universität ab: die biologischen Institute an der Siesmayerstraße (Botanik, Zoologie, Mikrobiologie, Anthropologie) platzten aus allen Nähten. Entlastung sollte der Umzug der Biologie an den neu entstehenden naturwissenschaftlichen Campus am Riedberg bei Niederursel bringen. Nach der Fertigstellung des „Biozentrum “ im Jahre 1994 zogen einige biologische Arbeitsgruppen an den Riedberg. An der Siesmayerstraße verblieben u. a. die Arbeitsgruppen, die den Botanischen Garten für Forschung und Lehre besonders intensiv nutzten. Fast zwei Jahrzehnte blieb der Fachbereich Biologie nun auf zwei Standorte verteilt (1994-2011). Dieser Zustand änderte sich erst mit dem Bezug des Biologicums und der Umsiedlung aller Arbeitsgruppen von der Siesmayerstraße an den Riedberg.

Die so entstandene räumliche Distanz von fast $10 \mathrm{~km}$ zwischen Botanischem Garten und 

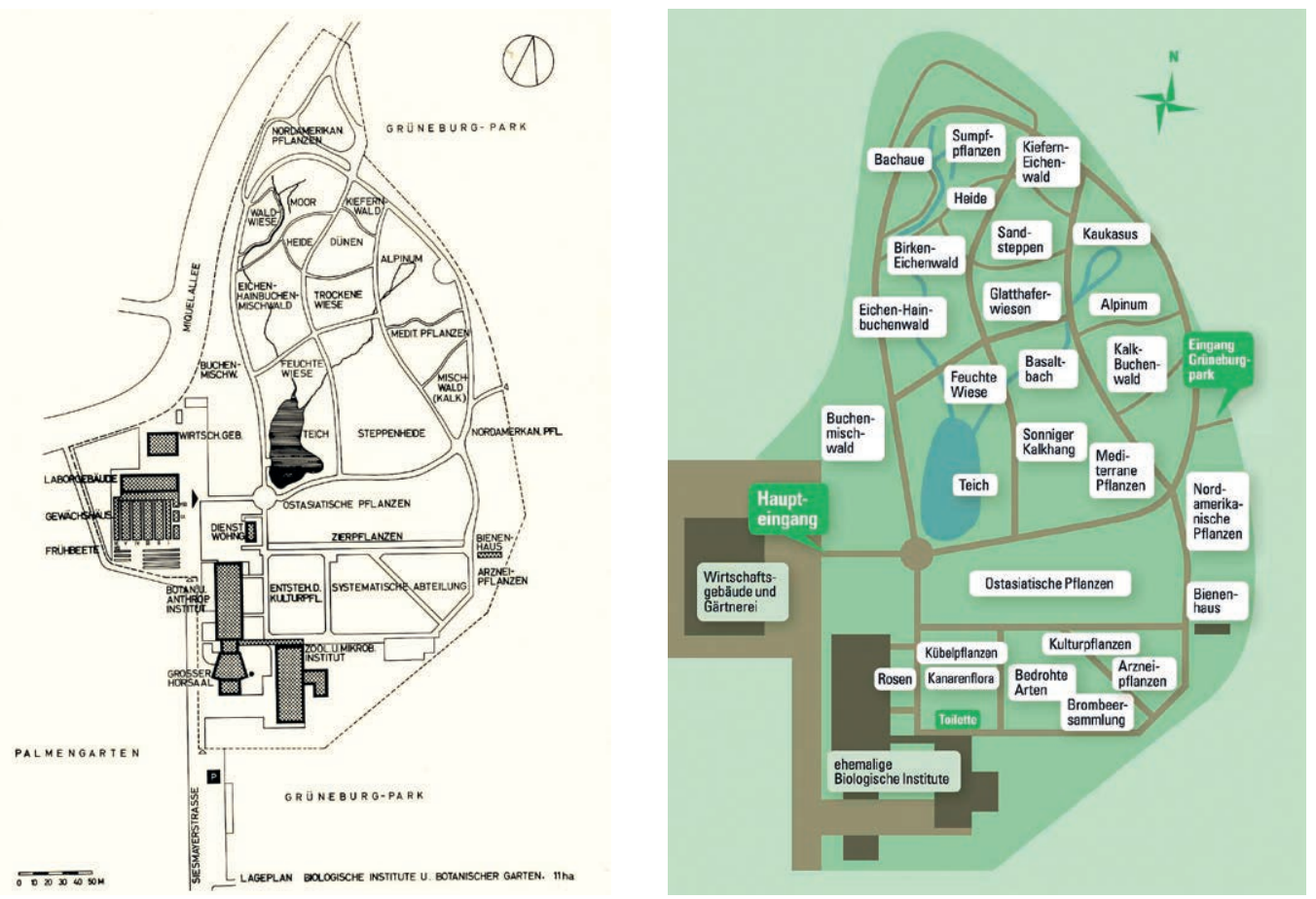

Abb. 11: Links: Plan des dritten Botanischen Gartens nach seiner Fertigstellung 1966 mit den Gewächshäusern und den damals neuen Gebäuden des Fachbereichs Biologie (EgLE \& Rosenstock 1966). Rechts: Aktueller Plan des Gartens (URL: https://www.botanischergarten-frankfurt.de/gartentour/geobotanische-abteilung/). Die zukünftige Nutzung der inzwischen stillgelegten Gebäude und des angrenzenden südlichsten Areals ist noch nicht geklärt.

naturwissenschaftlichen Instituten war mit den vielfältigen Anforderungen an den Garten nicht mehr zu vereinbaren. Daher entschloss sich die Universität, am Riedberg einen neuen, also den vierten Botanischen Garten in Frankfurt anzulegen. Damit stellte sich schon früh die Frage nach der Zukunft des Botanischen Gartens an der Siesmayerstraße und seines Areals. Der Universität war es nicht möglich, den Botanischen Garten an der Siesmayerstraße neben dem neuen Garten am Riedberg weiter zu betreiben. In der Jahre dauernden Diskussion um die Zukunft des Botanischen Gartens wurde eine breite Unterstützung in der Stadtgesellschaft für die Erhaltung dieses sowohl botanisch, naturschutzfachlich als auch ästhetischstädtebaulich einzigartigen Areals deutlich. Einen wichtigen Beitrag in der Klärung der Zukunftsfrage des Gartens leistete der „Freundeskreis des Botanischen Gartens e.V.“, der 2001 gegründet worden war. Ein wesentliches Ziel des Freundeskreises war die Erhaltung des Gartens am Standort Siesmayer- straße. Ein vom Forschungsinstitut Senckenberg entwickeltes Konzept zur Einbindung des Gartens in das Senckenberg Forschungsinstitut und Naturmuseum mit einer gleichzeitigen Erweiterung des wissenschaftlichen Bereiches wurde bei der Senckenberg-Evaluierung durch den Wissenschaftsrat 2005 vorgestellt, fand allerdings nicht die Zustimmung der Evaluierungskommission. Im Zusammenspiel von Stadt Frankfurt, Land Hessen und Goethe-Universität wurde dann schließlich eine Lösung vertraglich besiegelt, die den Fortbestand des gesamten Freiland-Areals des Botanischen Gartens und damit des schon immer öffentlich zugänglichen Teils sicherte. Lediglich der tropisch-subtropische Pflanzenbestand zog in die neuen Gewächshäuser am Riedberg um. Der Botanische Garten mit dem größten Teil seiner Mitarbeiter wurde Teil des Palmengartens und damit der Stadtverwaltung Frankfurt. Ein kleiner, südlicher Teil des früheren Areals mit den Gebäuden von ehemaligem Zoologischen und 


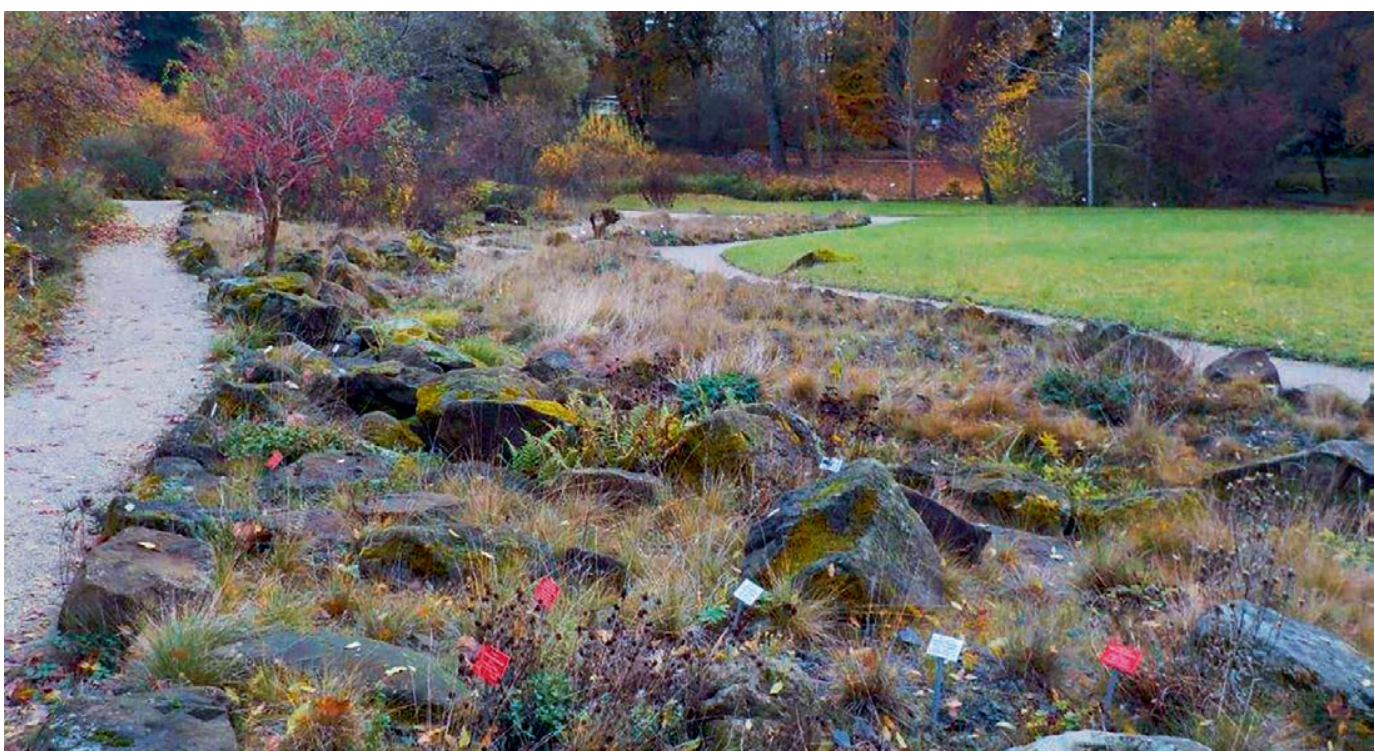

Abb. 12: Frühjahrsaspekt, Blick vom großen Teich nach Süden auf die Ostasienabteilung. (Foto: M. WesSEL)

Botanischen Institut verblieb im Besitz des Landes Hessen. Der endgültige Grenzverlauf zwischen städtischem und hessischem Gelände in unmittelbarer Nähe zu den alten Institutsgebäuden, ebenso wie deren Zukunft, ist noch ungeklärt. Die einzigartigen botanischen Anlagen werden seither in ihrer Artenvielfalt und Naturnähe erhalten und stehen der Öffentlichkeit mit Ausnahme der Wintermonate November bis Februar weiterhin kostenfrei zur Verfügung.

Was hat sich geändert? Die systematische Abteilung als typisch universitäres Lehr-Instrument sowie die Anlage mit Ruderalpflanzen wurden stillgelegt und durch gänzlich neu aufgebaute Anlagen ersetzt. Im Einzelnen befindet sich nun dort die Abteilung "Gefährdete Arten und Erhaltungskulturen" mit u. a. einem Hoch- und einem Kalkflachmoor, Beeten mit Heide-Arten, Arten auf kalkfreiem Sand sowie auf Kalkschotter. Die neophytischen Pflanzen sind genauso an prominentere Stelle umgezogen wie die alte Weinrebenund die Rubus-Sammlung und außerdem finden wir nun viele Pflanzen, die von Philipp Franz von Siebold in die Gartenkultur Europas eingeführt wurden. Intensiviert wurden die Aktivitäten im Bereich des Naturschutzes durch Projekte, die zur Erhaltung bedrohter hessischer Arten an den
Naturstandorten beitragen. Neu entstanden ist der Senckenbergische Arzneipflanzengarten, der 2007 anlässlich des 300. Geburtstags des Stifters eröffnet wurde. Hier fand ein bereits im ersten Botanischen Garten im Senckenbergianum am Eschenheimer Turm stehender Wassertrog nun seinen Platz. Das an eine breitere Öffentlichkeit gerichtete gärtnerisch-botanische Angebot des Gartens und seines Freundeskreises mit Ausstellungen, Führungen, Vorträgen und Informationstafeln im Garten ist kontinuierlich ausgebaut worden. Seit 2018 verhilft ein in der Kombination von Leitelementen, barrierefreier Beschilderung sowie einer App plus Audioguide einmaliges Projekt auch blinden und sehbehinderten Menschen zu Gartenerlebnissen. Weitere Angaben zur Geschichte finden sich u. a. bei Möbius (1893), Egle \& Rosenstock (1966), Conert (1967), Zizka (2001, 2005) und Stieglitz $(2011,2013,2014)$.

\section{Der vierte Botanische Garten - der Wissenschaftsgarten der GOETHE- Universität auf dem Campus Riedberg}

Die planerischen Vorarbeiten für einen neuen Botanischen Garten am Riedberg begannen bereits 1994, einige Jahre später legte Bucher (1998) ein Konzept im Rahmen einer von Prof. Dr. RüDiger WitTig betreuten Dissertation vor, das 
noch von einer Anlage schwerpunktmäßig nach pflanzensoziologischen Gesichtspunkten - in Teilen ähnlich dem dritten Garten - ausging. Sowohl aus Kostengründen, aber auch im Bemühen um Komplementarität der Gärten wurde der Anteil der sehr arbeitsintensiven pflanzensoziologischen Reviere stark reduziert. Erst ab Ende 2011 erfolgte schließlich der lang geplante Umzug der restlichen biologischen Arbeitsgruppen in das neu erbaute Biologicum am Campus Riedberg, 2013 wurde das neue Gewächshaus des Wissenschaftsgartens mit $1.182 \mathrm{~m}^{2}$ Kulturfläche fertiggestellt und die komplette Sammlung an Gewächshauspflanzen an den Riedberg umgezogen. Am 1.6.2014 wurde der Wissenschaftsgarten der Goethe-Universität offiziell eröffnet. Die insgesamt ca. 7 ha große Freilandfläche wird nach und nach entwickelt und umfasst bisher auf knapp 4 ha u. a. eine systematische Abteilung, einen Arzneipflanzengarten, den von der Biologie-Didaktik genutzten Bereich „Schule im Wissenschaftsgarten“, Streuobstbestände, Wiesen- und junge Waldbereiche sowie umfangreiche Versuchsflächen. Einen inhaltlichen Schwerpunkt stellen verschiedene Waldtypen dar, die naturgemäß z. Zt. noch in ganz frühen Stadien vorhanden oder noch in Planung sind.

\section{Ausblick}

Die heutigen drei Botanischen Gärten in Frankfurt (Palmengarten, Botanischer Garten an der Siesmayerstraße, Wissenschaftsgarten) nehmen unterschiedliche Aufgaben wahr, ergänzen sich inhaltlich und arbeiten eng zusammen, sie bilden gleichsam ein „Frankfurter Gartennetzwerk“. Der Palmengarten erfreut sich mit seinem breiten Angebot aus gärtnerischer und wissenschaftlicher Botanik und Kultur und einer der größten tropischen Pflanzensammlungen weltweit mit Abstand des größten öffentlichen Interesses. Sein Angebot, das im Freiland viele Kultursorten und Varietäten umfasst, wird durch die einzigartigen ökologischen und pflanzengeographischen Anlagen des angrenzenden Botanischen Gartens mit meist „reinen“ Arten bestens ergänzt. Während die wissenschaftliche Nutzung dort naturgemäß zurückgegangen ist, hat das Interesse der Öffentlichkeit zugenommen. Intensiviert wurden die Aktivitäten im Bereich des Artenschutzes mit den zahlreichen Erhaltungskulturen bedrohter Arten aus Hessen und Deutschland sowie die Umwelt- und Naturbildung. Dies sollen auch in Zukunft wichtige Arbeitsfelder bleiben. Der noch im Aufbau befindliche Wissenschaftsgarten am Riedberg erfüllt vor allem die

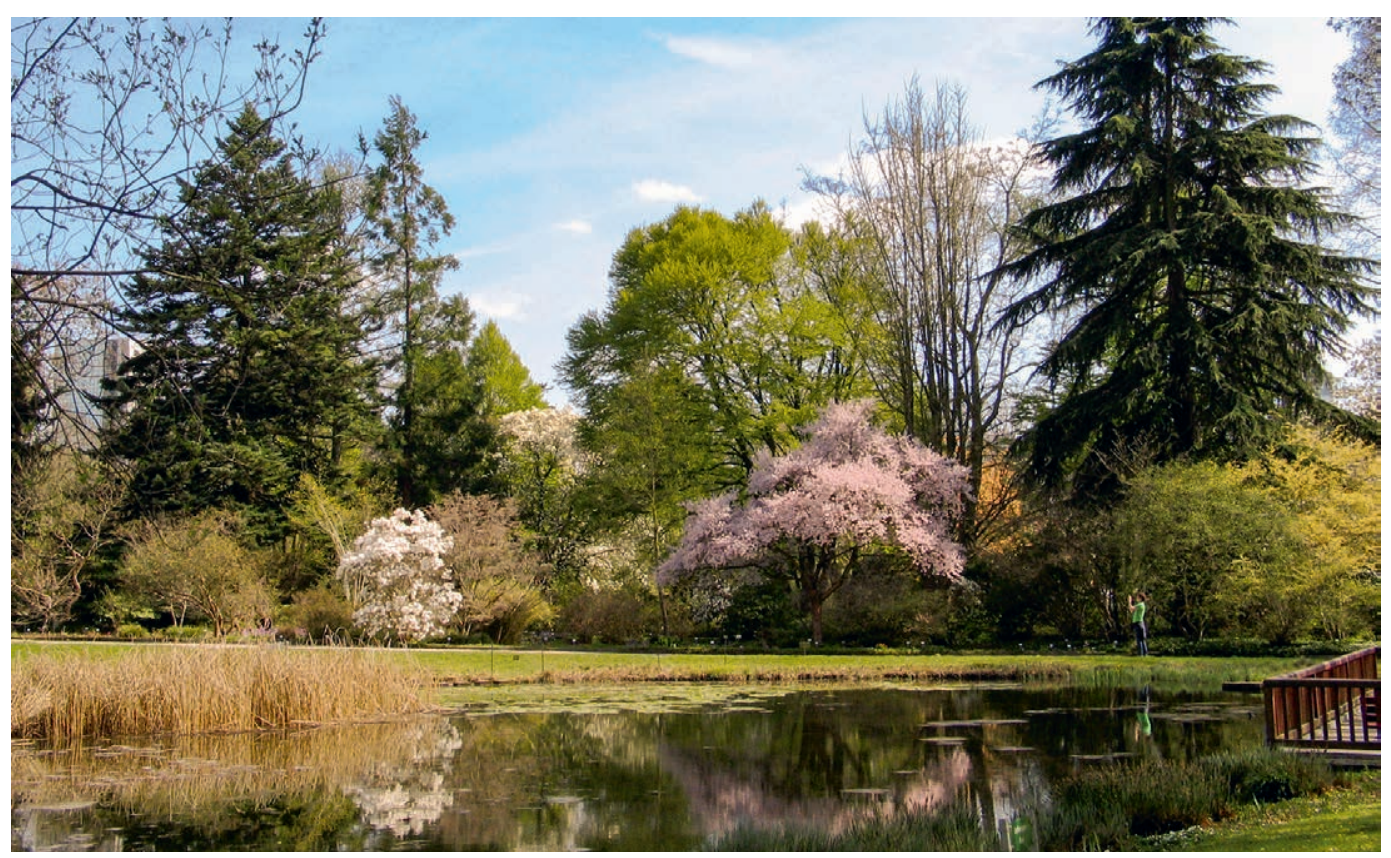

Abb. 13: Blick vom Alpinum nach Südwesten, im Vordergrund Pflanzen der Basaltmagerrasen. (Foto: M. Wessel) 
Aufgaben für universitäre Lehre und Forschung, gewinnt aber auch zunehmend Bedeutung als „grüne Oase“ des Campus und des Stadtteils. Für die mit rund 46000 Studenten drittgrößte Universität Deutschlands ist der Wissenschaftsgarten ein notwendiges Ausbildungs- und Forschungsinstrument. Beispiele für die enge Zusammenarbeit zwischen den Gärten sind z. B. die Planungen für eine gemeinsame Pflanzendatenbank, aber auch die Unterstützung des noch jungen Wissenschaftsgartens bei der Bereitstellung von Pflanzenmaterial durch Botanischen Garten und Palmengarten. Darüber hinaus gibt es Überlegungen, die wissenschaftliche Betreuung von Botanischem Garten und Wissenschaftsgarten stärker zu verbinden, bei dem auch die 2012 gegründete Adickes-Stiftung eine Rolle spielt (UniReport 2012).

Die drei Botanischen Gärten Frankfurts sind nicht nur notwendige, komplementäre Bildungsund Forschungseinrichtungen, sondern auch kulturell und geschichtlich wichtige Elemente der Stadt Frankfurt. Es sind Zentren der pflanzlichen Artenvielfalt die - neben ihren oben geschilderten „Fach“-Funktionen - auch einzigartige Leistungen im Bereich Naturerfahrung, Erholung und Ästhetik für die Bürger erbringen.

\section{Literatur}

Alberternst, B., Butterfass, T., Grasmück, H., LangeBertalot, H., Prinzinger, R., Trommer, G., Wessel, M., Wiltschкo, W., Winter, Ch., Wittig, R. \& Zizka, G. 2005: Der Botanische Garten der J.W. Goethe-Universität Frankfurt am Main: Ein illustrierter Führer. - 99 S.; BoD (Books on Demand), Norderstedt.

Blecken, F. 1994: Die Verpflanzung und Rettung der Frankfurter Eibe im Jahre 1907 - ein Rückblick im Jahr der Eibe 1994 Teil I. - Der Palmengarten 58: 140-147.

Blecken, F. 1995: Die Verpflanzung und Rettung der Frankfurter Eibe im Jahre 1907 - ein Rückblick im Jahr der Eibe 1994 Teil II. - Der Palmengarten 59: 17-29.

Bucher, M. 1998: Wissenschaftliche Grundlagen und Empfehlungen für die Anlage der pflanzensoziologischen Abteilung des neuen Botanischen Gartens der Johann Wolfgang GoetheUniversität in Frankfurt am Main. - Dissertation, Fachbereich Biologie, Frankfurt am Main.

Conert, H. J. 1967: Die Geschichte der Botanisch-Paläobotanischen Abteilung. - Senckenbergiana Biologica 48: 1-57.
Egle, K. \& Rosenstock, G. 1966: Die Geschichte der Botanik in Frankfurt am Main. -Frankfurt am Main.

Jenny, M., Wessel, M. \& Winter, C. 2014 (Hrsg.): Der Botanische Garten Frankfurt am Main. - BoD (Books on Demand), Norderstedt.

Mertens, R. 2014: Die Villa Grüneburg, das sogenannte Grüneburgschlösschen, in Frankfurt am Main. - In BRockhoff, E. \& Merk, H. (Hrsg.): Frankfurter Parkgeschichten, Gesellschaft für Frankfurter Geschichte. - Frankfurt am Main.

Möвıus, M. 1903: Geschichte und Beschreibung des Botanischen Gartens zu Frankfurt a.M. - Bericht der Senckenbergischen Naturforschenden Gesellschaft 1903: 117-154.

Reichard, J. J. 1782: Enumeratio Stirpium horti botanici Senkenbergiani, qui Francofurti ad Moenum est. - Frankfurt am Main.

Rittweger, F. 1905: Die ehemaligen Gebäulichkeiten der Dr. Senckenbergischen Stiftung gegr. den 18. August 1763. Frankfurt am Main.

Stieglitz, A. 2011: Der Botanische Garten Frankfurt am Main: Vom Stiftsgelände des Dr. Senckenberg bis zu seinem heutigen Standort im Westend. Festschrift zum 10-jährigen Bestehen des Freundeskreises des Botanischen Gartens. Frankfurt am Main.

Stieglitz, A. 2013: 250 Jahre Dr. Senckenbergische Stiftung. Vom Hortus medicus des Dr. Senckenberg zum Botanischen Garten der Stadt Frankfurt am Main. - Frankfurt am Main.

Stieglitz, A. 2014: Geschichte des Botanischen Gartens in Frankfurt am Main. - In: Jenny, M., Wessel, M. \& Winter, C. (Hrsg.): Der Botanische Garten Frankfurt am Main.- BoD (Books on Demand), Norderstedt.

UniReport 2012: Adickes-Stiftung der Stadt Frankfurt. UniReport 2: 6 .

Zizka, G., Billensteiner, H. \& Menk, H. 2001: Siebzig Jahre „Gesellschaft der Freunde des Palmengartens e.V.“. - Palmengarten 65: 103-117.

ZizKA, G. 2005: Geschichte des Botanischen Gartens.- In: Alberternst, B., Butterfass, T., Grasmück, H., LangeBertalot, H., Prinzinger, R., Trommer, G., Wessel, M., Wiltschko, W., Winter, Ch., Wittig, R. \& Zizka, G. 2005: Der Botanische Garten der J.W. Goethe-Universität Frankfurt am Main: Ein illustrierter Führer. - BoD (Books on Demand), Norderstedt.

\section{Anschrift der Autoren}

Prof. Dr. Georg Zizka und Dr. Stefan Dressler, Senckenberg Forschungsinstitut und Naturmuseum, Abteilung Botanik und Molekulare Evolutionsforschung, Senckenberganlage 25, 60325 Frankfurt am Main. E-Mail: georg.zizka@senckenberg.de bzw. stefan.dressler@senckenberg.de

Manfred Wessel, Palmengarten - Botanischer Garten, Siesmayerstraße 61, 60323 Frankfurt 